\title{
Indocyanine Green Nanoparticles for Theranostic Applications
}

\author{
Zonghai Sheng, Dehong Hu, Miaomiao Xue, Meng He, Ping Gong, Lintao Cai*
}

(Received 31 May 2013; accepted 19 June 2013; published online 25 July 2013)

\begin{abstract}
Theranostics is a concept that integrated imaging and therapy. As an emerging field, it embraces multiple techniques to arrive at an individualized treatment purpose. Indocyanine green (ICG) is a near infrared dye that has been approved by Food and Drug Administration (FDA) in USA for the use in indicator-dilution studies in humans. ICG nanoparticles (NPs) have attracted much attention for its potential applications in cancer theranostics. This review focuses on the preparation, application of ICG NPs for in vivo imaging (fluorescent imaging and photoacoustic imaging) and therapeutics (photothermal therapy, photodynamic therapy and photoacoustic therapy), and future directions based on recent developments in these areas. It is hoped that this review might provide new impetus to understand ICG NPs for cancer theranostics.
\end{abstract}

Keywords: Indocyanine green (ICG); Nanoparticles; Theranostics; Cancer

Citation: Zonghai Sheng, Dehong Hu, Miaomiao Xue, Meng He, Ping Gong and Lintao Cai, "Indocyanine Green Nanoparticles for Theranostic Applications", Nano-Micro Lett. 5(3), 145-150 (2013). http://dx.doi. org/10.5101/nml.v5i3.p145-150

\section{Introduction}

Theranostic is coined originally as a term to describe a treatment platform that combines diagnostics with therapeutics [1]. It embraces multiple techniques to toward the goal of personalized medicine for the benefit of patients. At present, a variety of nanoparticles (NPs) have been developed to diagnose and treat cancer. However, tumor heterogeneity and adaptive resistance remain formidable challenges to diagnosis and therapy [2]. Recently, there is an effort to tangle the emerging approach with nanotechnologies, in an attempt to develop theranostic NPs. As a nextgeneration nanocarrier system, theranostic NPs can not only provide patients with various treatment options, but also can monitor therapeutic efficacy following treatments. Therefore, it can expedite clinician's individualized therapeutic decisions [3]. At present, several NPs including quantum dots, magnetic NPs, carbon nanotubes, gold nanostructures, polymeric NPs, and silica NPs have been used as theranostic agents for cancer diagnosis and therapy [4]. However, little number of NPs has been approved by the Food and Drug Administration (FDA) in USA for clinical use as transport vehicles, and few theranostic NPs have entered human use.

Indocyanine green (ICG, molecular weight: 774.96 $\mathrm{g} / \mathrm{mol}$, CAS number: 3599-32-4) is an amphiphilic tricarbocyanine dye (Fig. 1) [5]. In 1958, ICG was approved by FDA for the use in indicator-dilution studies in humans, and it is also one of the least toxic agents ever administered to humans, with the only known adverse reaction being rare anaphylaxis. ICG exhibits emission maxima around $800 \mathrm{~nm}$ that make it highly suitable for bio-imaging applications with high signalto-background ratio (Fig. 1). It also can convert the absorbed light energy to produce heat and toxic chemical species (such as singlet oxygen, superoxide anions, and hydroxyl radicals), forming the respective bases for photoacoustic imaging (PA), photothermal (PT) and photodynamic (PD) therapies, respectively (Fig. 2) [5-

Guangdong Key Laboratory of Nanomedicine, CAS Key Laboratory of Health Informatics, Shenzhen Institutes of Advanced Technology, Chinese Academy of Sciences, Shenzhen 518055, China

*Corresponding author. E-mail: lt.cai@siat.ac.cn 


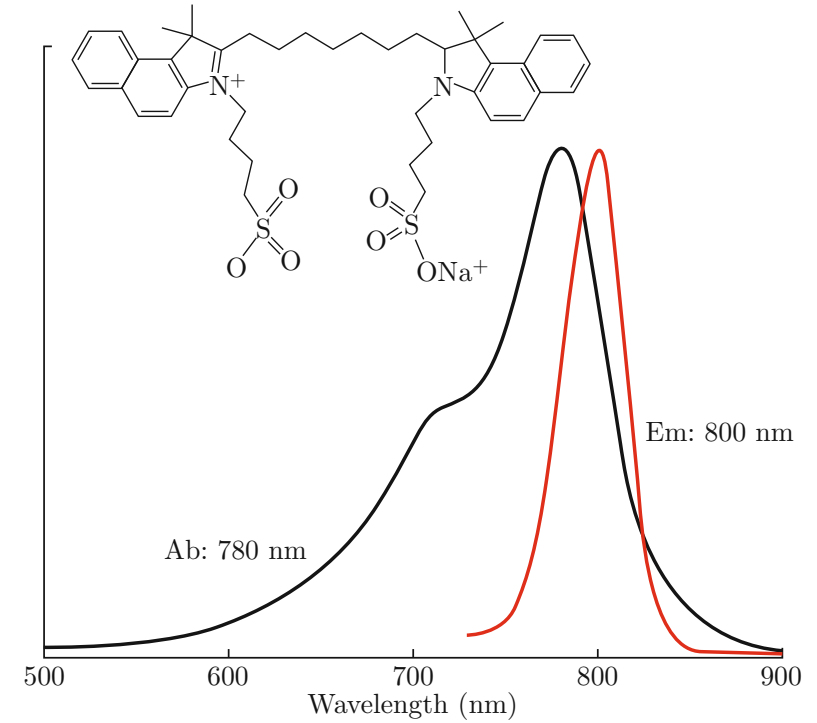

Fig. 1 The chemical structure, UV-Vis and fluorescent spectrum of ICG [5].

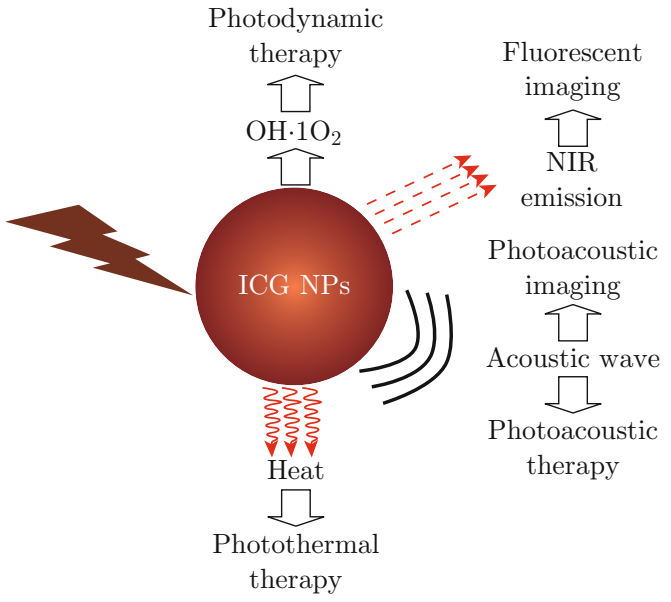

Fig. 2 The schematic diagram of ICG NPs for different applications.

8]. Therefore, ICG has been considered as a good theranostic agent. However, the use of ICG for imaging ther- apeutic applications is limited by its several drawbacks [9]. It is mainly manifested in the following aspects: 1) Photo-degradation: Degradation of ICG in aqueous solution followed first-order kinetics, and was accelerated by light exposure. 2) Thermal-degradation: Higher temperatures markedly accelerate the degradation of ICG in aqueous solutions. 3) Aqueous-instability: ICG undergoes degradation in aqueous media resulting in a simultaneous loss of absorption and fluorescence. 4) Short circulation time (half-life 2-4 min). 5) Easy to bind nonspecifically to human serum albumin and result in its rapid clearance by the liver. 6) Low fluorescence quantum yield due to internal conversion and photobleaching. Therefore, to overcome these limitations, ICG molecular can be loaded, doped and conjugated with different NPs with different preparing strategies (Fig. 3) [10]. The prepared ICG NPs have high photostability, thermal stability, long circulation time, and tumor-targeting ability [11], respectively.

In this article, we will review the various types of ICG NPs, and discuss their applications in cancer theranostics. A large number of review articles describe clinical diagnostic or therapeutic systems of ICG for the treatment of cancer, however, few articles have focused on ICG NPs capable of simultaneously imaging and treating cancer [12]. Thus, this review will focus on ICG NP that integrate tumor imaging and therapy into a single system. In detail, we will discuss theranostic applications of diverse ICG NPs categorized by different carriers such as silica, calcium phosphate, nanoGUMBOS, gold, PLGA, phospholipid-polyethylene glycol, PLGAlipid NPs, and so on (Table 1), or by different preparing strategies (Fig. 3). These applications include: 1) in vivo diagnostics of tumor; 2) photothermal therapy of tumor; 3) theranostics for monitoring biological responses and therapeutic efficacy following treatment. Finally, we will address the limitations and future challenges of ICG NPs.

Table 1 Theranostic applications of diverse ICG NPs categorized by different carriers.

\begin{tabular}{cccc}
\hline NPs & Imaging modalities & Therapeutics & \\
\hline Inorganic NPs & & & \\
\hline Silica NPs & NIRF, MRI & PTF & 28 \\
Calcium Phosphate NPs & NIRF & PTT & 30 \\
Nanoparticle-assembled capsules & NIRF & PTT & 25 \\
NanoGUMBOS & NIRF & PTT & 20 \\
\hline Organic NPs & & PTT \\
PLGA NPs & NIRF & PTT & 21 \\
Pluronic F-127 & NIRF & PTT & 18 \\
PLGA-lipid & NIRF & PIRF $/$ PA & \\
\hline
\end{tabular}




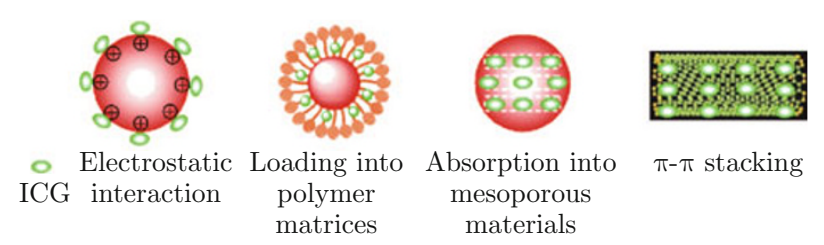

Fig. 3 Commonly used techniques to prepare ICG NPs.

\section{ICG loaded NPs}

As an amphiphilic dye, ICG can be loaded into several materials including calcium phosphosilicate [13], poly(allylamine hydrochloride) assembled mesocapsules [14], PLGA [15], PLGA-PEG [16], PL-PEG [17], Pluronic F-127 [18], $\mathrm{SiO}_{2}$ [19], ionic liquids [20], and so on. Among them, biodegradable-biocompatible, FDA approved PLGA carriers are one of the most common systems employed to improve ICG for theranostic applications [21]. ICG-PLAG NPs allowed passive targeting from the blood stream into the tumor site. In addition, after functionalized modification, targeting ligands can be conjugated to ICG-PLGA NPs to make them cancer specific. A good example is the PLGA-lipid NPs, reported by Zheng et al. in 2012. Using the single-step self-assemble and nanoprecipitation method, ICG can be loaded into the biodegradable PLGA-lipid micelles [22]. The average hydrodynamic diameter of the NPs is $102.4 \pm 4 \mathrm{~nm}$, which was quite stable without the size change for several weeks. Moreover, the ICG-PLGAlipid NPs exhibit good biocompatibility, monodispersity, significant stability against photobleaching and long circulation time. The targeting modality, folic acid (FA) ligand, can be functionalized onto the micelle surface to target the folate receptor found on tumors. On the basis of the results, the PLGA-lecithin-PEG NPs containing DOX and ICG were synthesized using a single-step sonication method, reported by Zheng et al. in 2013 [23]. The prepared theranostic nanoagents could simultaneously deliver DOX and ICG to tumor regions for combined chemo-photothermal therapy, and showed higher temperature response, faster DOX release under laser irradiation, and longer retention time in tumor. The combined treatment of ICG NPs with laser irradiation synergistically induced the apoptosis and death of MCF-7 and MCF-7/multidrug resistance cell, and suppressed tumor growth in vivo. Notably, no tumor recurrence was observed after only a single dose of NPs with laser irradiation. Hence, the welldefined theranostic ICG NPs exhibited great potential in targeting cancer imaging and chemo-photothermal therapy. Similarly, Zhong et al. reported phospholipidpolyethylene glycol (PL-PEG) NPs consisting of ICG. The photoacoustic therapy was investigated for the first time in vitro and in vivo [24]. When photoacoustic treatment was performed on mice, the laser and ICGPL-PEG-FA treatment caused significant tumor sup- pression.

In addition to PLAG, Wong and coworkers reported the synthesis of ICG-containing nano/microcapsules through a self-assembly chemistry [14]. Aqueous solutions of polyallylamine hydrochloride $(\mathrm{PAH})$ and dihydrogen phosphate salt are mixed together to form spherical aggregates. ICG was encapsulated into the NPs with electrostatic interactions. The reaction can be carried out under mild conditions, such as room temperature, aqueous solution, and neutral $\mathrm{pH}$. The particle size range is $0.6-1.0 \mu \mathrm{m}$, and the maximum loading is $23 \mathrm{wt} \%$. After that, Wong's group further investigated the ICG-nanocapsules, and developed three-step roomtemperature synthesis method for the preparation of $\sim 120 \mathrm{~nm}$ ICG-nanocapsules, and coated with antiepidermal growth factor receptor(anti-EGFR) antibodies for tumor cell targeting capability [25]. Photothermal effect of ICG-nanocapsules on EGFR-rich tumor cells was investigated. Significant thermal ablation of cancer cells was observed for encapsulated ICG as compared to free ICG at $808 \mathrm{~nm}$ laser irradiation with radiant exposure of $6 \mathrm{~W} / \mathrm{cm}^{2}$. This is the first demonstration of the theranostic ICG NPs for targeting fluorescence imaging and photothermal treatment of cancerous cells. Recently, Yaseen et al. further investigated how the surface coating and size of the ICG-nanocapsules influences ICG's biodistribution in vivo [26]. The results indicated that the nanocapsules can enhance the delivery of ICG to organs other than the liver, particularly the lung and spleen. Therefore, by manipulating synthetic parameters, the uptake of ICG-nanocapsules, in particular by the lung may potentially be controlled. It offers potential for the development of optically mediated diagnostic and treatment applications for these organs.

\section{ICG doped NPs}

As inorganic material, $\mathrm{SiO}_{2}$ exhibits excellent stability and biocompatibility. The FDA listed silica as "generally recognized as safe" [27]. ICG doped $\mathrm{SiO}_{2}$ NPs protect the ICG from photobleaching and photodegradation. The silica surface also enables easy and flexible surface treatments. Recently, Quan et al. successfully developed a method for preparation of ICG doped silica NPs [28]. Cationic polymer polyethylenimine (PEI) was interacted with anion ICG, and the ion-pair was encapsulated by a silica layer using the stöber method. The prepared ICG doped $\mathrm{SiO}_{2} \mathrm{NPs}$ have diameters in the range $50-200 \mathrm{~nm}$. The isolation of ICG molecules from the surroundings by the silica layer greatly enhanced the photostability of ICG doped $\mathrm{SiO}_{2}$ NPs. However, in vivo imaging and therapy ability of the ICG doped $\mathrm{SiO}_{2}$ NPs have not been investigated. Lee et al. reported ICG doped mesoporous silica NPs for optical imaging in living mice for the 
first time [29]. The high dispersion of ICG molecules in the large surface areas of mesoporous silica could efficiently prevent them from aggregation and thus decrease the fluorescence self-quenching. In addition, the nanochannels of mesoporous silica provided the confined space not only can protect ICG molecules from the irreversible degradation but also diminish the immune response. The studies of in vivo biodistribution of ICG doped mesoporous silica revealed that the particles in the size of $50-100 \mathrm{~nm}$ possessing relatively positive surface charge were mainly targeting to the liver. In addition to $\mathrm{SiO}_{2}$, ICG also can be doped into $\mathrm{Ca}_{3} \mathrm{P}_{2}$ NPs, a primary biomineral with good biocompatibility and biodegradation, using a double reverse micelle technique [30]. After polyethylene glycol (PEG) surface functionality, ICG-Ca $\mathrm{P}_{2} \mathrm{NPs}$ exhibit higher fluorescent intensity, quantum efficiency and photostability than the free ICG. The in vivo imaging indicated that the ICG-Ca $\mathrm{P}_{2}$ NPs can be accumulated in solid, xenograft breast adenocarcinoma tumors via enhanced retention and permeability (EPR) within $24 \mathrm{~h}$ after tail vein injection in a nude mouse model.

\section{ICG conjugated NPs}

Chemical conjugate is an ideal way for the preparation of ICG NPs [31]. However, it is not easy to introduce active groups on the ICG molecular due to its symmetry of chemical structure. Therefore, this strategy is not generally used. Recently, two derivatives of ICG, ICG-Sulfo-OSu and azide-terminated ICG, attracted much attentions. Mok et al. reported the use of ICG-Sulfo-OSu for the preparation of ICG-nanogel [32]. The carboxyl groups of hyaluronic acid (HA) were activated via carbodiimide coupling chemistry and reacted with ethylenediamine to prepare amine modified hyaluronic acid $\left(\mathrm{NH}_{2}-\mathrm{HA}\right)$. Subsequently, the ICG-Sulfo-OSu was reacted with $\mathrm{NH}_{2}-\mathrm{HA}$, and formed ICG encapsulated HA. The fluorescence of ICG-nanogel is self-quenched due to its strong intermolecular hydrophobic interactions between ICGs. Under the HAdase-overexpressing conditions, such as tumor microenvironments and metastatic lymph nodes, the fluorescence of ICG-nanogel were recovered. As a theranostic agent, the ICG-nanogel can be accumulated specifically in tumor after intravenous injection, and enable visualization of tumors. Moreover, the ICG fluorescence can last three days after the injection. Therefore, we could monitor biological responses and therapeutic efficacy following treatment using ICG-nanogels. In addition to using the EDC coupling reactions, click chemistry, a highly efficient conjugation reaction, is also used for the synthesis of ICG conjugated NPs. Foulger group reported the synthesis of azide-terminated ICG by four steps of chemical reaction [33]. The ICG was conjugated with poly(propargyl acrylate) (PA) NPs with copper-catalyzed azide/alkyne cycloaddition. The placement of ICG onto the surface of the particles allows for the chromophore to complex with proteins that resulted in the alteration and enhancement of the emission of the dye. Moreover, PDT studies indicated the ICG-PA NPs with $780 \mathrm{~nm}$ radiation resulted in a statistically significant reduction in cell growth. Based on the results of these studies, Daniele et al. further developed protein-activated ICG-magnetic nanoclusters [34]. The magnetic core could provide a MRI signal and ICG-BSA shell show a highly fluorescent imaging ability. Therefore, this system presents the blueprint for the next generation of multimodal imaging theranostic platforms.

\section{Conclusions}

In the current article, we have highlighted some ICG NPs that are currently under intensive investigation for theranostic applications. These ICG NPs can not only possess unique optical or photoacoustic imaging properties, but also can be used as therapeutic agents for cancer therapy. The clinical demand of cancer imaging and therapy has also led many researchers to turn to this field. However, despite the fast progress, there have so far been few ICG NPs that are so developed to meet clinical standards. Therefore, we still have a long way to go in terms of using these nano-systems in humans. The major questions include the following: 1) the biocompatibility and biodegradable nature of nanocarriers. 2) The low fluorescence quantum yield. 3) The low photothermal conversion efficiency. Furthermore, highly specific targeting is the other important topic that can never be over addressed. Therefore, much effort to address each of these issues is going on, and should remain the focus in the future studies.

\section{Acknowledgements}

The authors gratefully acknowledge support for this research from the National Natural Science Foundation of China (Grant No. 81071249, 81171446 and 20905050), Guangdong Innovation Team of Low-cost Healthcare, Science and Technology Key Project of Guangdong (2009A030301010) and Shenzhen (CXB201005250029A, JC201005270326A, JC201005260247A, JC201104220242A).

\section{References}

[1] X. Y. Chen, S. S. Gambhir and J. Cheon, "Theranostic nanomedicine", Acc. Chem. Res. 44(10), 841-841 (2011). http://dx.doi.org/10.1021/ar200231d

[2] K. Y. Choi, G. Liu, S. Lee and X. Y. Chen, "Theranostic nanoplatforms for simultaneous cancer imaging and therapy: current approaches and future perspec- 
tives", Nanoscale 4, 330-342 (2012). http://dx.doi. org/10.1039/c1nr11277e

[3] T. Lammers, S. Aime, W. E. Hennink, G. Storm and F. Kiessling, "Theranostic Nanomedicine", Acc. Chem. Res. 44(10), 1029-1038 (2011). http://dx.doi.org/ 10.1021/ar200019c

[4] J. V. Jokerst and S. S. Gambhir, "Molecular Imaging with Theranostic Nanoparticles", Acc Chem Res 44(10), 1050-1060 (2011). http://dx.doi.org/10. $1021 / \operatorname{ar} 200106 \mathrm{e}$

[5] W. S. Kuo, Y. T. Chang, K. C. Cho, K. C. Chiu, C. H. Lien, C. S. Yeh and S. J. Chen, "Gold nanomaterials conjugated with indocyanine green for dual-modality photodynamic and photothermal therapy", Biomaterials 33(11), 3270-3278 (2012). http://dx.doi.org/10. 1016/j.biomaterials. 2012.01.035

[6] B. M. Barth, E. I. Altinoglu, S. S. Shanmugavelandy, J. M. Kaiser, D. Crespo-Gonzalez, N. A. DiVittore, C. McGovern, T. M. Goff, N. R. Keasey, J. H. Adair, T. P. Loughran, Jr., D. F. Claxton and M. Kester, "Targeted indocyanine-green-loaded calcium phosphosilicate nanoparticles for in vivo photodynamic therapy of leukemia", Acs Nano 5(7), 5325-5337 (2011). http://dx.doi.org/10.1021/nn2005766

[7] G. Kim, S. W. Huang, K. C. Day, M. O'Donnell, R. R. Agayan, M. A. Day, R. Kopelman and S. Ashkenazi, "Indocyanine-green-embedded PEBBLEs as a contrast agent for photoacoustic imaging", J. Biomed. Opt. 12(4), 044020 (2007). http://dx.doi.org/10.1117/ 1. 2771530

[8] X. Zheng, D. Xing, F. Zhou, B. Wu and W. R. Chen, "Indocyanine green-containing nanostructure as near infrared dual-functional targeting probes for optical imaging and photothermal therapy", Mol. Pharm. 8(2), 447-456 (2011). http://dx.doi.org/10.1021/ mp100301t

[9] V. Saxena, M. Sadoqi and J. Shao, "Enhanced photostability, thermal-stability and aqueous-stability of indocyanine green in polymeric nanoparticulate systems", J. Photochem. Photobiol. B 74(1), 29-38 (2004). http://dx.doi.org/10.1016/j.jphotobiol. 2004.01 .002

[10] L. Larush and S. Magdassi, "Formation of nearinfrared fluorescent nanoparticles for medical imaging", Nanomedicine 6(2), 233-240 (2011). http://dx. doi.org/10.2217/nnm.11.5

[11] A. J. Gomes, L. O. Lunardi, J. M. Marchetti, C. N. Lunardi and A. C. Tedesco, "Indocyanine green nanoparticles useful for photomedicine", Photomedicine and Laser Surgery 24(4), 514-521 (2006). http://dx.doi. org/10.1089/pho.2006.24.514

[12] D. Grosenick, H. Wabnitz and B. Ebert, "Recent advances in contrast-enhanced near infrared diffuse optical imaging of diseases using indocyanine green", J. Near Infrared Spec. 20(1), 203-221 (2012). http://dx . doi.org/10.1255/jnirs. 964

[13] B. M. Barth, R. Sharma, E. I. Altinoglu, T. T. Morgan, S. S. Shanmugavelandy, J. M. Kaiser, C. McGovern, G. L. Matters, J. P. Smith, M. Kester and J. H. Adair, "Bioconjugation of calcium phosphosilicate composite nanoparticles for selective targeting of human breast and pancreatic cancers in vivo", Acs Nano 4(3), 12791287 (2010). http://dx.doi.org/10.1021/nn901297q

[14] J. Yu, M. A. Yaseen, B. Anvari and M. S. Wong, "Synthesis of near-infrared-absorbing nanoparticleassembled capsules", Chem. Mater. 19(6), 1277-1284 (2007). http://dx.doi.org/10.1021/cm062080x

[15] V. Saxena, M. Sadoqi and J. Shao, "Indocyanine green-loaded biodegradable nanoparticles: preparation, physicochemical characterization and in vitro release", Int. J. Pharm. 278(2), 293-301 (2004). http:// dx.doi.org/10.1016/j.ijpharm.2004.03.032

[16] R. H. Patel, A. S. Wadajkar, N. L. Patel, V. C. Kavuri, K. T. Nguyen and H. Liu, "Multifunctionality of indocyanine green-loaded biodegradable nanoparticles for enhanced optical imaging and hyperthermia intervention of cancer", J. Biomed. Opt. 17(4), 046003 (2012). http://dx.doi.org/10.1117/1.JB0.17.4.046003

[17] X. Zheng, D. Xing, F. Zhou, B. Wu and W. R. Chen, "Indocyanine green-containing nanostructure as near infrared dual-functional targeting probes for optical imaging and photothermal therapy", Mol. Pharm. 8(2), 447-456 (2011). http://dx.doi.org/10.1021/ mp100301t

[18] H. Kim, Y. Chen, C. W. Mount, W. R. Gombotz, X. Li and S. H. Pun, "Evaluation of temperaturesensitive, indocyanine green-encapsulating micelles for noninvasive near-infrared tumor imaging", Pharm. Res. 27(9), 1900-1913 (2010). http://dx.doi.org/10. 1007/s11095-010-0190-y

[19] G. Kim, S. W. Huang, K. C. Day, M. O'Donnell, R. R. Agayan, M. A. Day, R. Kopelman and S. Ashkenazi, "Indocyanine-green-embedded PEBBLEs as a contrast agent for photoacoustic imaging", J. Biomed. Opt. 12(4), 044020 (2007). http://dx.doi.org/10.1117/ 1. 2771530

[20] D. K. Bwambok, B. El-Zahab, S. K. Challa, M. Li, L. Chandler, G. A. Baker and I. M. Warner, "Near-infrared fluorescent nanoGUMBOS for biomedical imaging", Acs Nano 3(12), 3854-3860 (2009). http://dx.doi.org/10.1021/nn9010126

[21] B. Bahmani, S. Gupta, S. Upadhyayula, V. I. Vullev and B. Anvari, "Effect of polyethylene glycol coatings on uptake of indocyanine green loaded nanocapsules by human spleen macrophages in vitro", J. Biomed. Opt. 16(5), 051303 (2011). http://dx.doi.org/10. 1117/1. 3574761

[22] C. F. Zheng, M. B. Zheng, P. Gong, D. X. Jia, P. F. Zhang, B. H. Shi, Z. H. Sheng, Y. F. Ma and L. T. Cai, "Indocyanine green-loaded biodegradable tumor targeting nanoprobes for in vitro and in vivo imaging", Biomaterials 33(22), 5603-5609 (2012). http:// dx.doi.org/10.1016/j.biomaterials. 2012.04.044

[23] M. Zheng, C. Yue, Y. Ma, P. Gong, P. Zhao, C. Zheng, Z. Sheng, P. Zhang, Z. Wang and L. Cai, "Single-step assembly of DOX-ICG loaded lipid-polymer nanoparticles for highly effective chemo-photothermal combination therapy", Acs Nano 7(3), 2056-2067 (2013). http://dx.doi.org/10.1021/nn400334y 
[24] J. Zhong, S. Yang, X. Zheng, T. Zhou and D. Xing, "In vivo photoacoustic therapy with cancertargeted indocyanine green-containing nanoparticles", Nanomedicine 8(6), 903-919 (2013). http://dx.doi. org/10.2217/NNM.12.123

[25] J. Yu, D. Javier, M. A. Yaseen, N. Nitin, R. Richards-Kortum, B. Anvari and M. S. Wong, "Selfassembly synthesis, tumor cell targeting, and photothermal capabilities of antibody-coated indocyanine green nanocapsules", J. Am. Chem. Soc. 132(6), 19291938 (2010). http://dx.doi.org/10.1021/ja908139y

[26] M. A. Yaseen, J. Yu, B. Jung, M. S. Wong and B. Anvari, "Biodistribution of encapsulated indocyanine green in healthy mice", Mol. Pharm. 6(5), 1321-1332 (2009). http://dx.doi.org/10.1021/mp800270t

[27] P. Sharma, N. E. Bengtsson, G. A. Walter, H. B. Sohn, G. Zhou, N. Iwakuma, H. Zeng, S. R. Grobmyer, E. W. Scott and B. M. Moudgil, "Adoliniumdoped silica nanoparticles encapsulating indocyanine green for near infrared and magnetic resonance imaging", Small 8(18), 2856-2868 (2012). http://dx.doi. org/10.1002/smll. 201200258

[28] B. Quan, K. Choi, Y. H. Kim, K. W. Kang and D. S. Chung, "Near infrared dye indocyanine green doped silica nanoparticles for biological imaging", Talanta 99, 387-393 (2012). http://dx.doi.org/10.1016/j . talanta.2012.05.069

[29] C. H. Lee, S. H. Cheng, Y. J. Wang, Y. C. Chen, N. T. Chen, J. Souris, C. T. Chen, C. Y. Mou, C. S. Yang and L. W. Lo, "Near-infrared mesoporous silica nanoparticles for optical imaging: characterization and in vivo biodistribution", Adv. Funct. Mater.
19(2), 215-222 (2009). http://dx.doi.org/10.1002/ adfm. 200800753

[30] E. I. Altinoglu, T. J. Russin, J. M. Kaiser, B. M. Barth, P. C. Eklund, M. Kester and J. H. Adair, "Nearinfrared emitting fluorophore-doped calcium phosphate nanoparticles for in vivo imaging of human breast cancer", Acs Nano 2(10), 2075-2084 (2008). http://dx.doi.org/10.1021/nn800448r

[31] K. Sano, T. Nakajima, K. Miyazaki, Y. Ohuchi, T. Ikegami, P. L. Choyke and H. Kobayashi, "Short PEGlinkers improve the performance of targeted, activatable monoclonal antibody-indocyanine green optical imaging probes", Bioconjug. Chem. 24(5), 811-816 (2013). http://dx.doi.org/10.1021/bc400050k

[32] H. Mok, H. Jeong, S. J. Kim and B. H. Chung, "Indocyanine green encapsulated nanogels for hyaluronidase activatable and selective near infrared imaging of tumors and lymph nodes", Chem. Commun. (Camb) 48, 8628-8630 (2012). http://dx.doi.org/10.1039/ c2cc33555g

[33] P. Rungta, Y. P. Bandera, R. D. Roeder, Y. Li, W. S. Baldwin, D. Sharma, M. G. Sehorn, I. Luzinov and S. H. Foulger, "Selective imaging and killing of cancer cells with protein-activated near-infrared fluorescing nanoparticles", Macromol. Biosci. 11(7), 927-937 (2011). http://dx.doi.org/10.1002/mabi. 201100043

[34] M. A. Daniele, M. L. Shaughnessy, R. Roeder, A. Childress, Y. P. Bandera and S. Foulger, "Magnetic nanoclusters exhibiting protein-activated near-infrared fluorescence", Acs Nano 7(1), 203-213 (2013). http:// dx.doi.org/10.1021/nn3037368 